\title{
Electronic structure of graphene under periodic uniaxial tensile strain
}

\author{
Hikari Tomori, ${ }^{1,}$ a) Mina Maruyama, ${ }^{1, b)}$ and Susumu Okada ${ }^{1, c)}$ \\ Department of Physics, Graduate School of Pure and Applied Sciences, \\ University of Tsukuba, 1-1-1 Tennodai, Tsukuba, Ibaraki 305-8571, \\ Japan
}

Using density functional theory with the generalized gradient approximation, we investigate the electronic structure of graphene with uniaxial tensile strain in terms of its direction and homogeneity. A uniform strain does not affect the conical dispersion band of pristine graphene but simply shifts its position in the two-dimensional Brillouin zone. Graphene with periodic tensile strain along the armchair and chiral directions is a semiconductor with a tiny gap of a few $\mathrm{meV}$, which is slightly dependent on the strain direction.

\footnotetext{
a) E-mail address: tomori4486@gmail.com

b) E-mail address: mmaruyama@comas.frsc.tsukuba.ac.jp

c)E-mail address: sokada@comas.frsc.tsukuba.ac.jp
} 


\section{INTRODUCTION}

Ever since its exfoliation and synthesis ${ }^{1-5}$, graphene has maintained a premier position in fundamental and applied sciences, because of its unique structural and electronic properties $^{6,7}$. A honeycomb network of $\mathrm{sp}^{2}$ carbon atoms renders conical dispersion bands at the K point and the Fermi level, which make it a superior material possessing unusual electronic properties $^{8-10}$. The conical dispersion bands cause a massless electron that is free from scattering, which leads to a remarkable carrier mobility of up to a few hundred thousand $\mathrm{cm}^{2} / \mathrm{Vs}^{11-13}$. Despite its high carrier mobility, it is difficult to fabricate semiconducting devices using graphene as a channel material, owing to the absence of a band gap. Structural modulation and hybrid structures occasionally open a finite band gap on graphene, which enables them to be used as constituent materials for semiconducting switching devices, even if such derivatives cause a deterioration of the carrier mobility owing to the edges and borders. Graphene nanoribbons ${ }^{14-16}$ and nanomeshes ${ }^{17-22}$ are representative semiconducting graphene derivatives, whose band gap strongly depends on their sizes and edge atomic arrangements. The formation of in-plane heterostructures with hexagonal boron nitride (h$\mathrm{BN}$ ) also results in semiconducting electronic structures of the resultant hybrids comprising graphene and h-BN ${ }^{23-25}$. Bilayer graphene under an external electric field ${ }^{26-29}$ and van der Waals heterostructures with other two-dimensional materials ${ }^{30}$ also have a finite bad gap which depends on their geometric structures and external conditions.

In addition to the above-mentioned graphene derivatives, mechanical strains also open the band gap of graphene without introducing edges and borders. Local strain causes a pseudo magnetic field that causes asymmetry in its sublattices, which causes a finite gap between bonding and antibonding $\pi$ bands $^{31-34}$. Furthermore, experiments have reported that graphene under tensile strains exhibits semiconducting electronic properties. ${ }^{35,36}$ For instance, experiments have shown that graphene with a non-uniform strain possesses an energy gap between bonding and antibonding $\pi$ states, which implies the presence of the corresponding pseudomgnetic field. Although the gap formation has clearly been observed in experiments, little is known about the microscopic insight into the electronic structures of graphene with uniaxial tensile strain in terms of their direction. Accordingly, we aim to investigate the electronic structure of graphene with uniaxial strains along the lattice orientations of $0,10.9,19.1$, and $30^{\circ}$, using density functional theory (DFT) with the generalized 
(a)

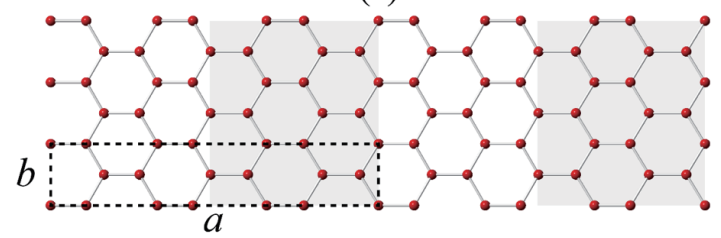

(b)

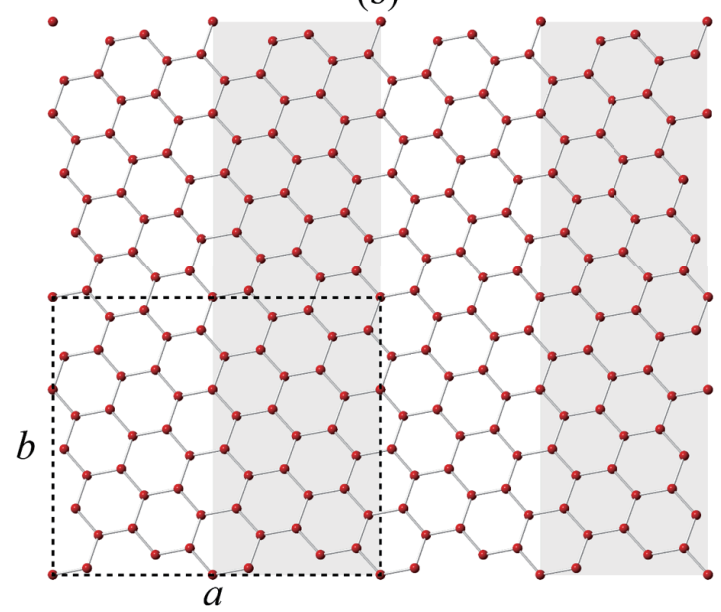

(c)

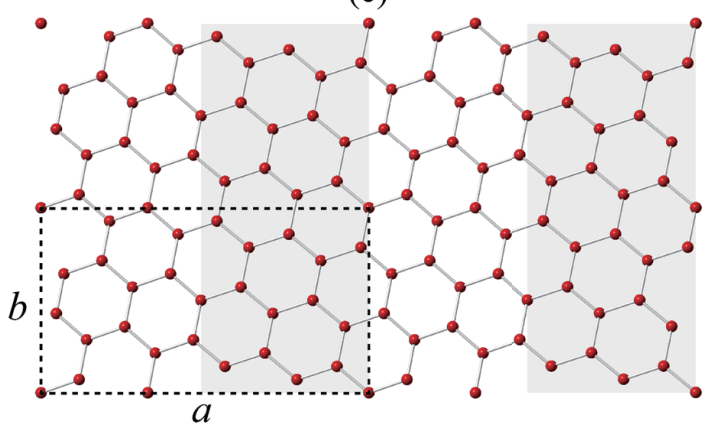

(d)

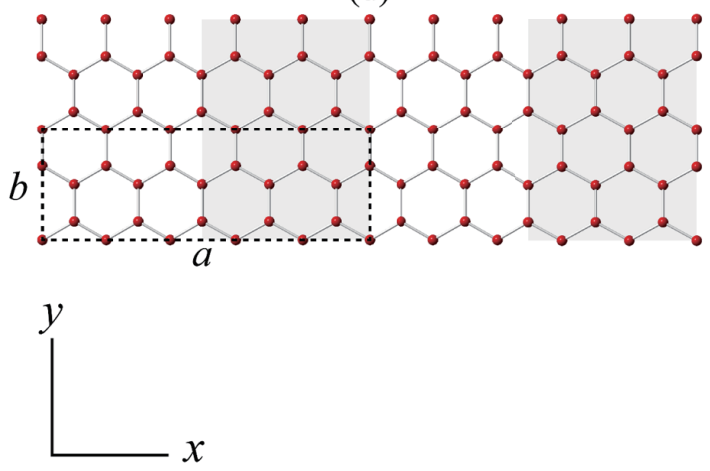

FIG. 1. Geometric structures of graphene with periodic uniaxial strain along the (a) $0^{\circ}$ (armchair), (b) $10.9^{\circ}$, (c) $19.1^{\circ}$, and (d) $30^{\circ}$ (zigzag) directions. Strains are applied along the $x$ axis in each figure. The shaded area indicates the graphene with a uniaxial tensile strain of $5 \%$. Dashed rectangles indicate the unit cell.

gradient approximation (GGA). Calculations revealed that the uniform tensile strain does not cause a band gap irrespective of the tensile direction. In contrast, nonuniform strain along the armchair and chiral directions caused a small but finite band gap owing to the symmetry breaking at the border between the strained and unstrained domains.

\section{CALCULATION METHODS AND STRUCTURAL MODELS}

We used DFT ${ }^{37,38}$ implemented in a STATE package ${ }^{39}$ to calculate the self-consistent electronic structure of strained graphene. An exchange-correlation potential energy between interacting electrons was treated using the GGA with the Perdew-Burke-Ernzerhof functional form ${ }^{40,41}$. An ultrasoft pseudopotential was used to describe the interaction between 
TABLE I. Lattice parameters $(a, b)$ of rectangular cells of strained graphene, where $a$ corresponds with the strain directions of $0^{\circ}$ (armchair), $10.9^{\circ}, 19.1^{\circ}$, and $30^{\circ}$ (zigzag).

\begin{tabular}{ccccc}
\hline \hline & $0^{\circ}$ & $10.9^{\circ}$ & $19.1^{\circ}$ & $30^{\circ}$ \\
\hline Uniform strain $(\AA)$ & $(13.42,2.45)$ & $(13.66,11.27)$ & $(11.83,6.51)$ & $(12.91,4.26)$ \\
Periodic strain $(\AA)$ & $(13.10,2.45)$ & $(13.34,11.27)$ & $(11.55,6.51)$ & $(12.61,4.26)$ \\
\hline \hline
\end{tabular}

electrons and ions ${ }^{42}$. Valence wave functions and deficit charge density were expanded by the plane-wave basis set with cutoff energies of 25 and $225 \mathrm{Ry}$, respectively. To investigate the electronic structure of strained graphene in terms of their strain direction, we applied a tensile strain in the directions of $0,10.9,19.1$, and $30^{\circ}$ with respect to the armchair direction. To apply uniaxial strain, we considered a rectangular cell with cell parameters that were the same as the above strain directions (Fig. 1). Lattice parameters for strained graphene are summarized in Table I. Graphene with nonuniform strain was simulated by a superlattice consisting of pristine and strained graphene strips that were alternately arranged.For the strained graphene, graphene was elongated by $5 \%$ along the strain direction. The Brillouinzone integration was performed with $2 \times 8 \times 1,2 \times 2 \times 1,2 \times 3 \times 1$, and $2 \times 4 \times 1 \boldsymbol{k}$-meshes for selfconsistent electronic structure calculations of the rectangular cells of graphene with strain directions of $0^{\circ}$ (armchair), $10.9^{\circ}, 19.1^{\circ}$, and $30^{\circ}$ (zigzag), respectively, which gave sufficient convergence of the total energy and electronic structure of graphene and its derivatives.

\section{RESULTS AND DISCUSSION}

Figure 2 shows the electronic energy band of graphene with uniform tensile strain in directions of $0,10.9,19.1$, and $30^{\circ}$. The strain along the armchair direction slightly shifted the Dirac point to $\mathrm{J}_{y}$ point along the $\Gamma-\mathrm{J}_{y}$ line. However, the strain did not open the band gap between bonding and antibonding $\pi$ states [a left figure in Fig. 2(a)]. In the case, these states perfectly touch each other at the Dirac point (Table II). For the tensile strain along chiral directions, the strains also shifted the Dirac point but it did not affect the metallic electronic structure [left figures in Figs. 2(b) and 2(c)]. Note that our calculations could not give numerical accuracy in eigenvalues of less than $1 \mathrm{meV}$, although these two have a finite gap value of less than $1 \mathrm{meV}$ (Table II). The tensile strain along the zigzag direction 
(a)
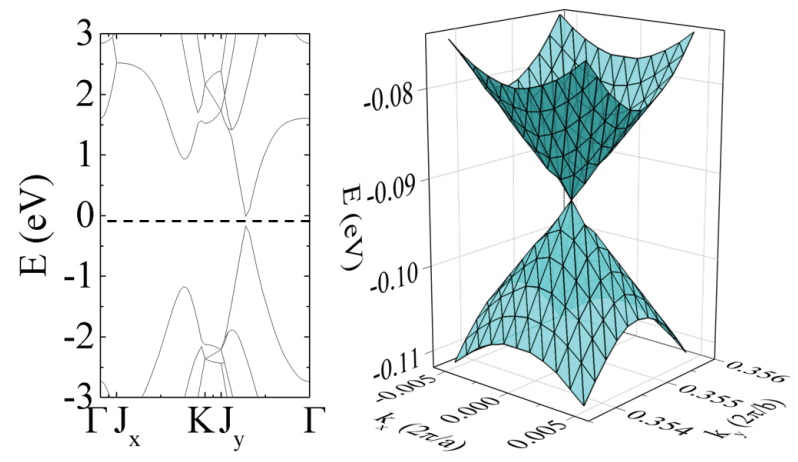

(b)
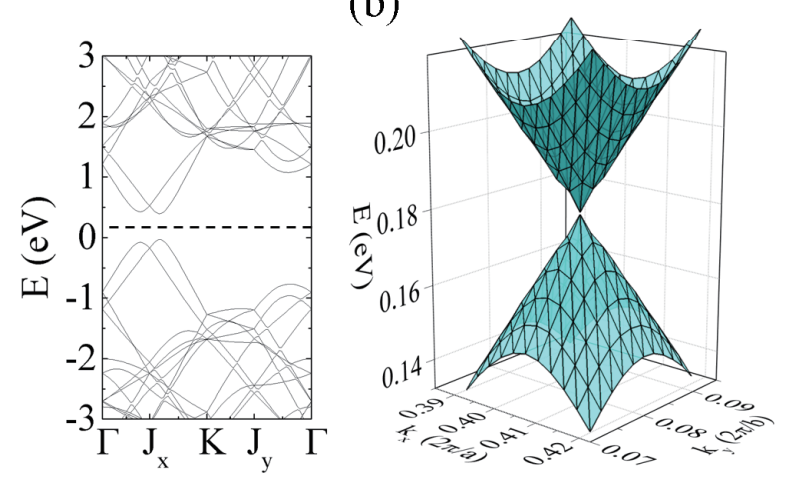

(c)
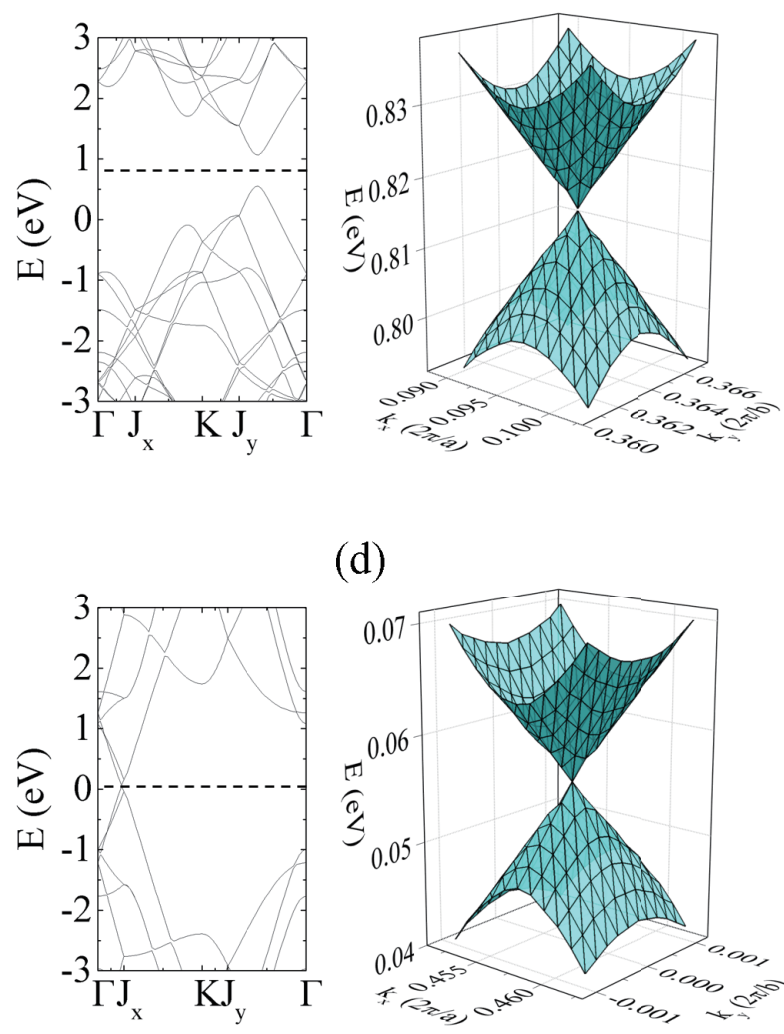

(d)

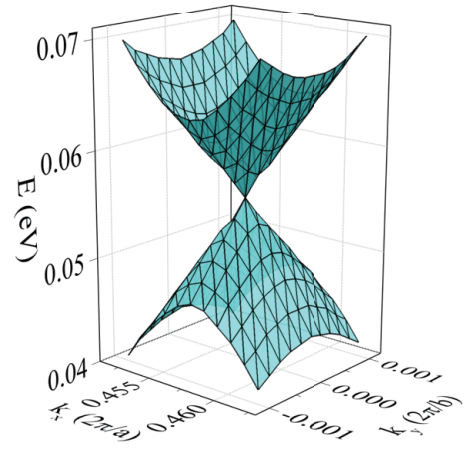

FIG. 2. Electronic energy band along the high symmetry points in the rectangular Brillouin zone (left in each figure) and bird's-eye views of the highest occupied and lowest unoccupied branches at the vicinity of the Dirac cone (right in each figure) of graphene with uniform uniaxial strain along the (a) $0^{\circ}$ (armchair), (b) $10.9^{\circ}$, (c) $19.1^{\circ}$, and (d) $30^{\circ}$ (zigzag) directions. The energy is measured from that of the Dirac point. The horizontal dotted line in each left figure indicates the Fermi level energy. For the bird's-eye views, $11 \times 11$ fine mesh around the Dirac point is taken for investigating the tiny gap between bonding and antibonding $\pi$ states.

shifted the Dirac point to the $\Gamma$ point along the $\Gamma-J_{x}$ line, and did not open the band gap. Therefore, the uniform tensile strain is unlikely to modulate the characteristic band structure of graphene as reported in previous manuscripts ${ }^{43-45}$. Indeed, the wave functions of the Dirac cone were homogeneously distributed on the graphene irrespective of the tensile directions (Fig. 3)

Next, we investigated the electronic structure of strained graphene in which the strained and pristine graphene strips were alternately arranged. For simplicity, we considered a sharp 
TABLE II. Calculated energy gap between the top of valence band and the bottom of conduction band of graphene under uniform and periodic uniaxial strain. The parenthesis indicates the Dirac point in the Brillouin zone in $2 \pi / a$ and $2 \pi / b$ unit where $a$ and $b$ are the cell parameters of each rectangular cell.

\begin{tabular}{lcccc}
\hline \hline & $0^{\circ}$ & $10.9^{\circ}$ & $19.1^{\circ}$ & $30^{\circ}$ \\
\hline Uniform strain (meV) & 0.0 & 0.6 & 0.3 & 0.0 \\
& $(0.0000,0.3456)$ & $(0.4044,0.0825)$ & $(0.0959,0.3640)$ & $(0.4572,0.0000)$ \\
Periodic strain (meV) & 3.5 & 1.4 & 4.1 & 0.6 \\
& $(0.0000,0.3443)$ & $(0.367,0.0043)$ & $(0.0475,0.3500)$ & $(0.3974,0.0000)$ \\
\hline \hline
\end{tabular}

border between the strained and unstrained regions. Figure 4 shows the electronic structure of graphene containing periodic tensile strain along the armchair, chiral, and zigzag directions. For the strain along the armchair direction, the Dirac point was slightly shifted towards to the $\Gamma$ point from the initial point at $2 / 3$ of the $\Gamma-J_{y}$ line [Fig. 4(a)]. By carefully investigating the band dispersion relation around the Dirac point, we found that the graphene with periodic strain possessed a tiny but finite band gap of $3.5 \mathrm{meV}$ between the bonding and antibonding $\pi$ states. For graphene with an periodic strain along $10.9^{\circ}$, we found the gap between the bonding and antibonding $\pi$ states along the $\Gamma-J_{x}$ line, which indicated that the Dirac point was dislodged from the $\Gamma-\mathrm{J}_{x}$ line. A bird's-eye view of the highest occupied and the lowest unoccupied bands indicated that the strained graphene had a small gap of $1.4 \mathrm{meV}$ at the middle of the $\Gamma-\mathrm{J}_{x}$ line with a small $k_{y}$ component. The periodically strained graphene with the strain direction of $19.1^{\circ}$ also exhibited a semiconducting electronic property with a gap of $4.1 \mathrm{meV}$ near the middle of the $\Gamma-\mathrm{J}_{y}$ line. Note that the calculated gap energies are underestimated compared with those obtained by more sophisticated methods, owing to the choice of the GGA functional in this work ${ }^{46,47}$. Thus, wider gap is expected to observed in experiments. In contrast to these strain directions, graphene with a periodic strain along the zigzag direction exhibited a peculiar behavior. The strained graphene still retained its metallic nature with the Dirac cone near the 2/3 of the $\Gamma-\mathrm{J}_{x}$ line.

The gap formation in strained graphene where the periodic strain directions were 0, 10.9, and $19.1^{\circ}$ was ascribed to the symmetry breaking at the zigzag border between the strained 
(a)

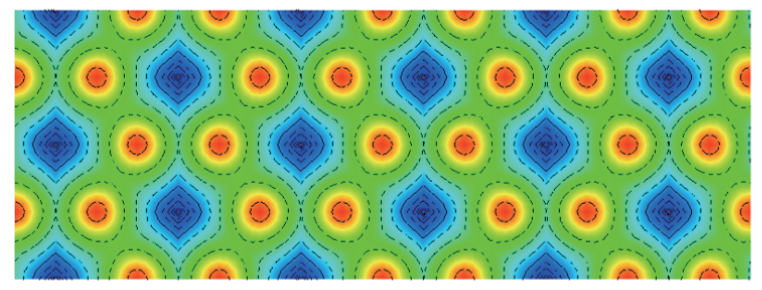

(b)
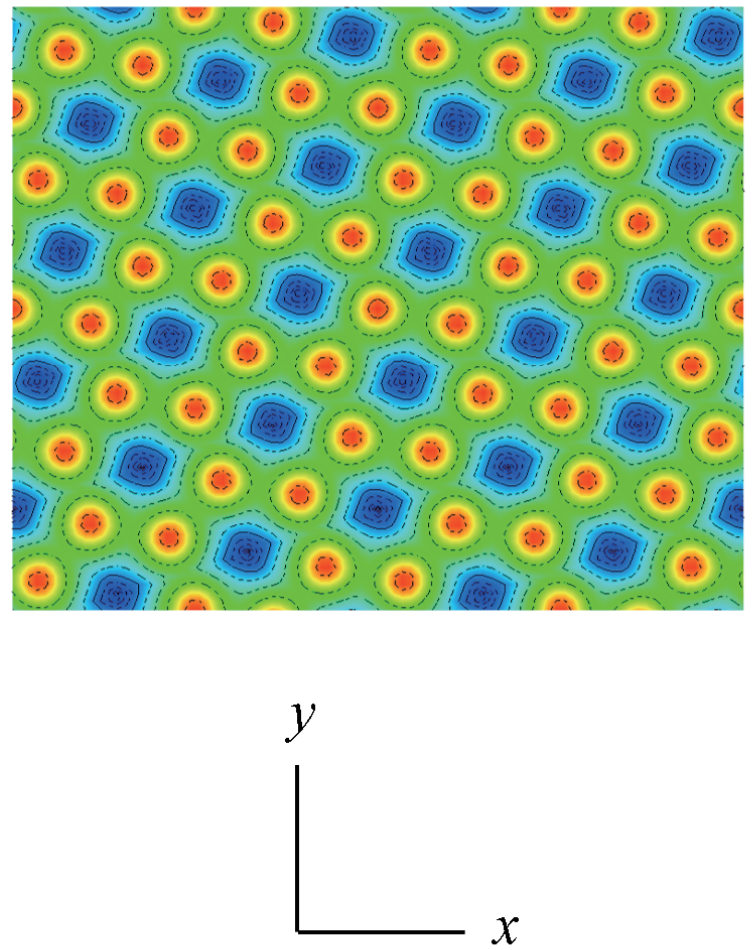

(c)

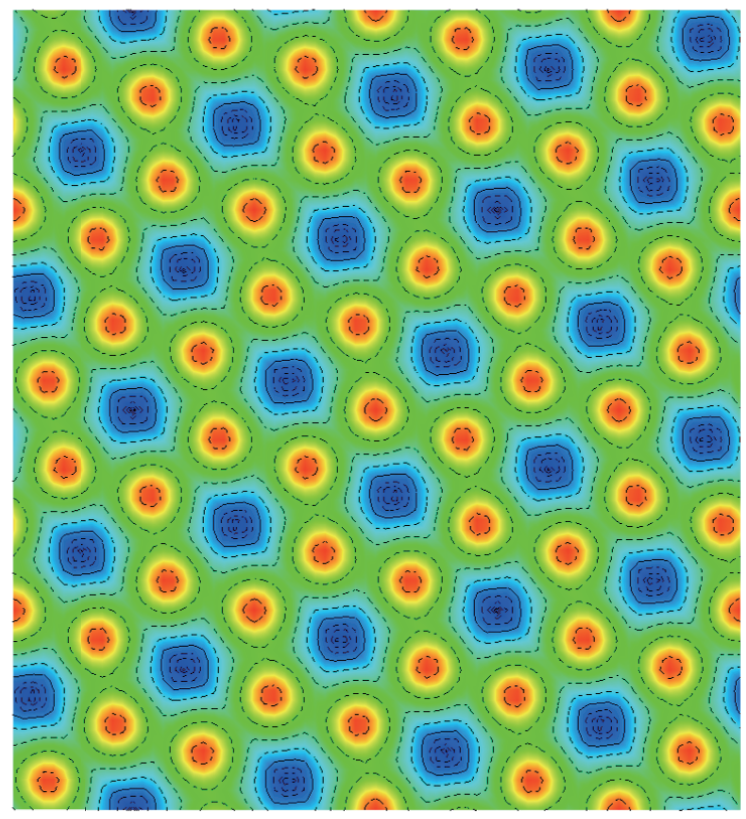

(d)

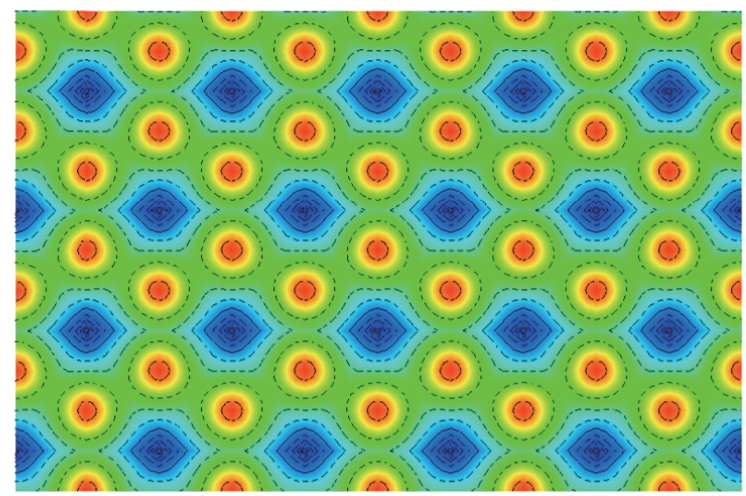

FIG. 3. Contour and color plots of squared wave function of the Dirac point of graphene with uniform uniaxial strain along the (a) $0^{\circ}$ (armchair), (b) $10.9^{\circ}$, (c) $19.1^{\circ}$, and (d) $30^{\circ}$ (zigzag) directions. Red, green, and blue regions correspond to the high, medium, and low charge density regions, respectively.

and pristine graphene strips. At the zigzag border, the symmetrical charge distribution in a $\mathrm{C}_{2}$ dimer was disrupted for all cases (Fig. 5), owing to the electrostatic potential modulation by the bond elongation. In the graphene with a periodic strain along the zigzag direction, the valence charge density retained its distribution in the strained and pristine strips at their 
(a)
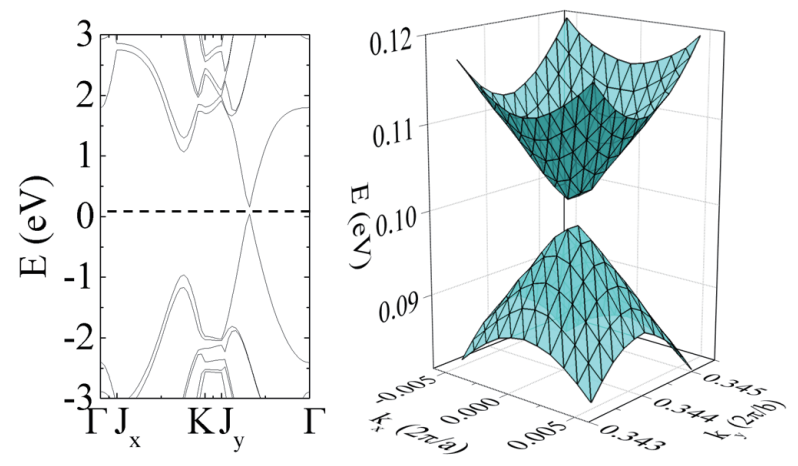

(b)
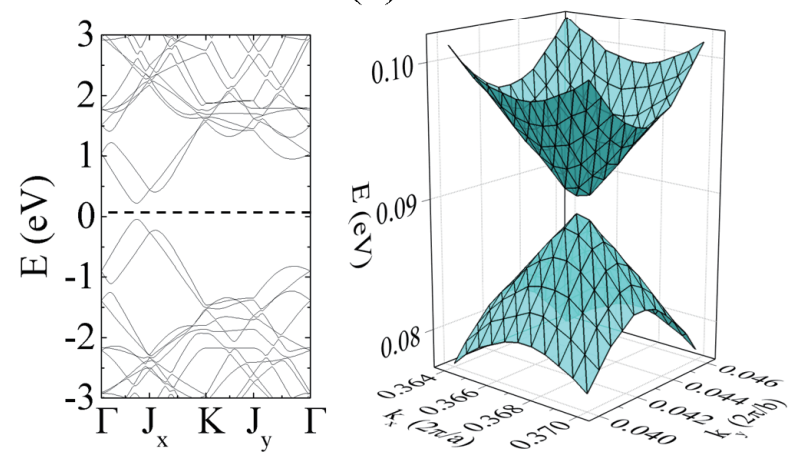

(c)
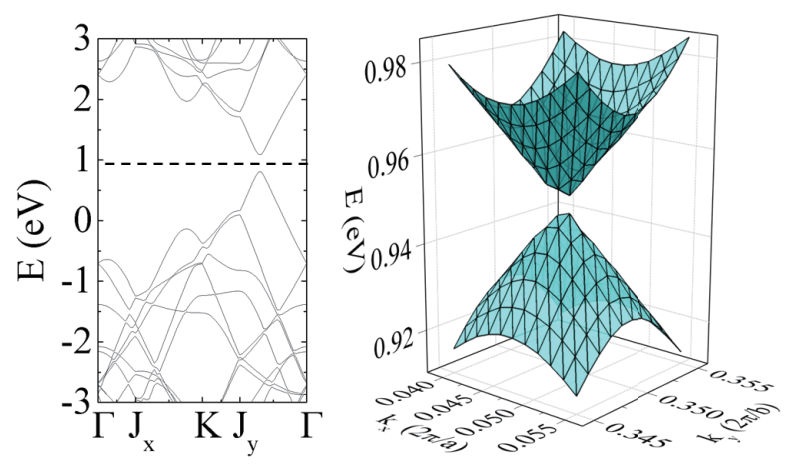

(d)

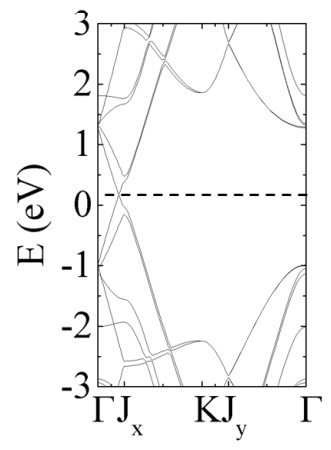

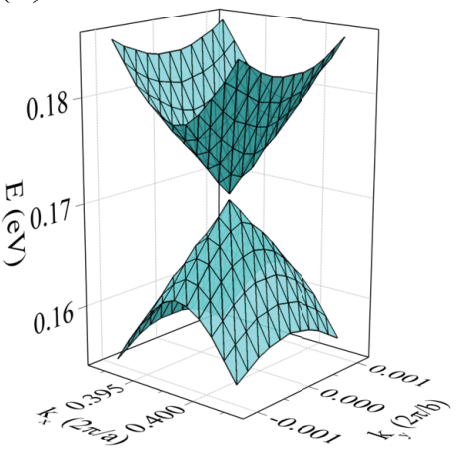

FIG. 4. Electronic energy band along the high symmetry points in the rectangular Brillouin zone (left in each figure) and bird's-eye views of the highest occupied and lowest unoccupied branches at the vicinity of the Dirac cone (right in each figure) of graphene with periodic uniaxial strain along the (a) $0^{\circ}$ (armchair), (b) $10.9^{\circ}$, (c) $19.1^{\circ}$, and (d) $30^{\circ}$ (zigzag) directions. The horizontal dotted line in each left figure indicates the Fermi level energy. For the bird's-eye views, $11 \times 11$ fine mesh around the Dirac point is taken for investigating the tiny gap between bonding and antibonding $\pi$ states.

zigzag border.

\section{CONCLUSION}

Based on DFT with GGA, the electronic structure of graphene with uniaxial strain was studied in terms of its direction. Our calculation revealed that graphene with uniform tensile strain retains its zero-gap electronic structure irrespective of the strain direction. 
(a)

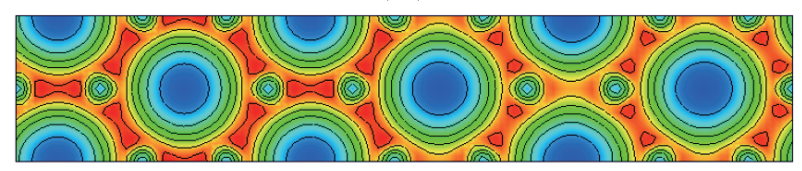

(b)

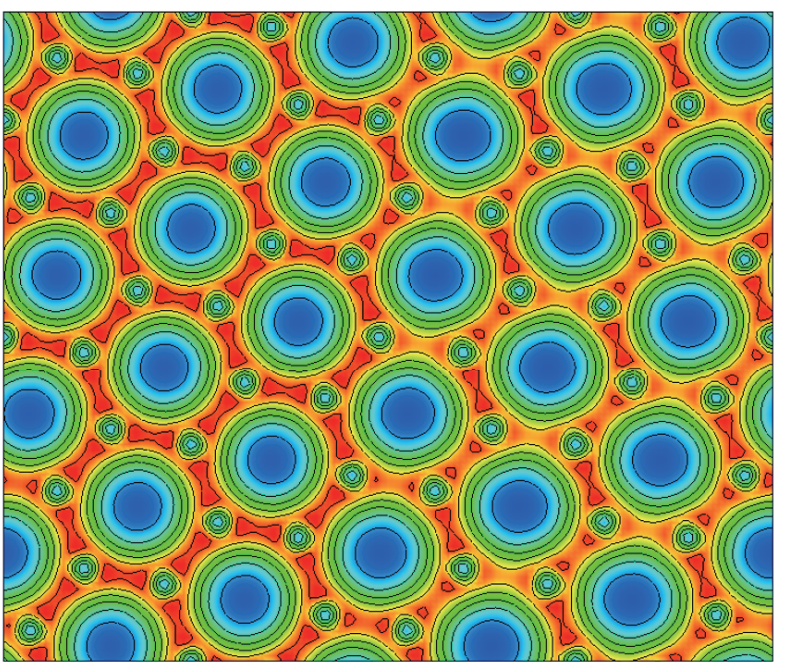

(c)

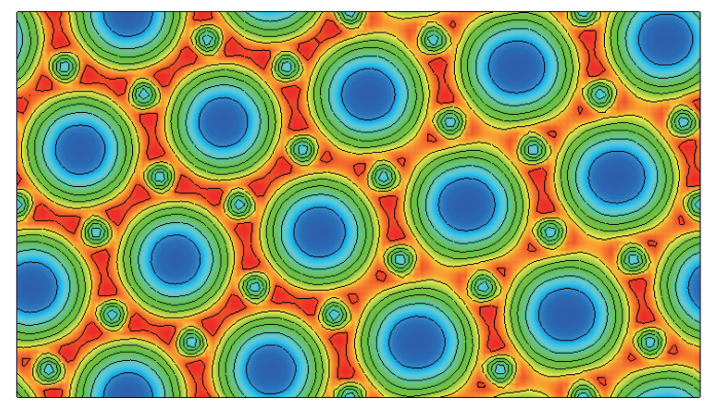

(d)
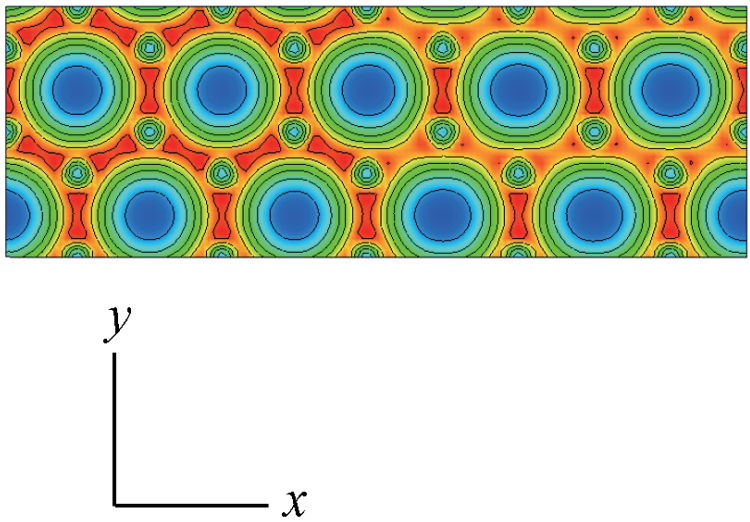

FIG. 5. Contour and color plots of valence charge density of graphene with periodic uniaxial strain along the (a) $0^{\circ}$ (armchair), (b) $10.9^{\circ}$, (c) $19.1^{\circ}$, and (d) $30^{\circ}$ (zigzag) directions. Red, green, and blue regions correspond to the high, medium, and low charge density regions, respectively.

The periodic strain, which is simulated by considering the two-dimensional superlattice comprising pristine and strained graphene strips, leads to the modulation of the electronic energy band at the Fermi level, and this depends on the strain direction. The periodic strain along the armchair and chiral directions opens a small but finite energy gap between the bonding and antibonding $\pi$ states, which leads to the semiconducting electronic structures. The gap formation in the strained graphene is ascribed to the symmetry breaking of the bipertite network at the zigzag border between pristine and strained graphene strips. In contrast, the periodic strain along the zigzag direction, owing to the armchair border between the pristine and strained graphene strips, still preserves the conical dispersion bands at the Fermi level, which leads to zero-gap semiconducting properties. For all cases, the uniform 
and periodic strain causes a shift of the Dirac point or the maximum/minimum of the valence/conduction bands, respectively, from that observed for the pristine graphene.

\section{ACKNOWLEDGMENTS}

The author thanks JST-CREST Grant Numbers JPMJCR1532 and JPMJCR1715 from the Japan Science and Technology Agency, JSPS KAKENHI Grant Numbers JP20K05253, JP20H02080, JP20H00316, JP17H01069, and JP16H06331 from the Japan Society for the Promotion of Science, the Joint Research Program on Zero-Emission Energy Research, Institute of Advanced Energy, Kyoto University, and University of Tsukuba Basic Reseach Support Program (S). Part of the calculations was performed on an NEC SX-Ace at the Cybermedia Center at Osaka University.

\section{REFERENCES}

${ }^{1}$ K. S. Novoselov, A. K. Geim, S. V. Morozov, D. Jiang, Y. Zhang, S. V. Dubonos, I. V. Grigorieva, and A. A. Firsov, Science 306, 666 (2004).

${ }^{2}$ A. K. Geim and K. S. Novoselov, Nat. Mater. 6, 183 (2007).

${ }^{3}$ I. Forbeaux, J.-M. Themlin, and J.-M. Debever, Phys. Rev. B 58, 16396 (1998).

${ }^{4}$ C. Berger, Z. Song, X. Li, X. Wu, N. Brown, C. Naud, D. Mayou, T. Li, J. Hass, A. N. Marchenkov, E. H. Conrad, P. N. First, and W. A. de Heer, Science 312, 1191 (2006).

${ }^{5}$ X. Li, W. Cai, J. An, S. Kim, J. Nah, D. Yang, R. Piner, A. Velamakanni, I. Jung, E. Tutuc, S. K. Banerjee, L. Colombo, and R. S. Ruoff, Science 324, 1312 (2009).

${ }^{6}$ M. S. Dresselhaus and G. Dresselhaus, Adv. Phys. 30, 139 (1981).

${ }^{7}$ A. H. Castro Neto, F. Guinea, N. M. R. Peres, K. S. Novoselov, and A. K. Geim, Rev. Mod. Phys. 81, 109 (2009).

${ }^{8}$ G. S. Painter and D. E. Ellis, Phys. Rev. 1, 4747 (1970).

${ }^{9}$ F. Bassani and G.P. Parravicini, Nuovo Cimento B 50, 95 (1967).

${ }^{10}$ M. Posternak, A. Baldereschi, A. J. Freeman, E. Wimmer and M. Weinert, Phys. Rev. Lett. 50, 761 (1983).

${ }^{11}$ K. I. Bolotin, K. J. Sikes, Z. Jiang, M. Klima, G. Fudenberg, J. Hone, P. Kim, and H. L. Stormer, Solid State Commun. 146, 351 (2008). 
${ }^{12}$ J. B. Oostinga, H. B. Heersche, X. Liu, A. F. Morpurgo, and L. M. K. Vandersypen, Nat. Mater. 7, 151 (2007).

${ }^{13}$ Y. Zhang, T. Tang, C. Girit, Z. Hao, M. C. Martin, A. Zettl, M. F. Crommie, Y. R. Shen, and F. Wang, Nature 459, 820 (2009).

${ }^{14}$ M. Fujita, K. Wakabayashi, K. Nakada, and K. Kusakabe, J. Phys. Soc. Jpn. 65, 1920 (1996).

${ }^{15}$ K. Nakada, M. Fujita, G. Dresselhaus, and M. S. Dresselhaus, Phys. Rev. B 54, 17954 (1996).

${ }^{16}$ Y.-W. Son, M. L. Cohen, and S. G. Louie, Phys. Rev. Lett. 97, 216803 (2006).

${ }^{17}$ T. Matsumoto and S. Saito, J. Phys. Soc. Jpn. 71, 2765 (2002).

${ }^{18}$ M. Sakurai, Y. Sakai, and S. Saito, J. Phys. Conf. Ser. 302, 012018 (2011).

${ }^{19}$ T. G. Pedersen, C. Flindt, J. Pedersen, A.-P. Jauho, N. A. Mortensen, and K. Pedersen, Phys. Rev. B 77, 245431 (2008).

${ }^{20}$ W. Liu, Z. F. Wang, Q. W. Shi, J. Yang, and F. Liu, Phys. Rev. B 80, 233405 (2009).

${ }^{21}$ H. Jippo, M. Ohfuchi, and C. Kaneta, Phys. Rev. B 84, 075467 (2011).

${ }^{22}$ M. Maruyama, N. T. Cuong, and S. Okada, Carbon 109, 755 (2016).

${ }^{23}$ S. Okada, M. Igami, K. Nakada, and A. Oshiyama, Phys. Rev. B 62, 9896 (2000).

${ }^{24}$ S. Okada and A. Oshiyama, Phys. Rev. Lett. 87, 146803 (2001).

${ }^{25}$ M. Maruyama and S. Okada, J. Phys. Chem. C 120, 1293 (2016).

${ }^{26}$ J. B. Oostinga, H. B. Heersche, X. Liu, A. F. Morpurgo, and L. M. K. Vandersypen, Nat. Mater. 7, 151 (2008).

${ }^{27}$ Y. Zhang, T.-T. Tang, C. Girit, Z. Hao, M. C. Martin, A. Zettl, M. F. Crommie, Y. R. Shen, and F. Wang, Nat. Mater. 459, 820 (2009).

${ }^{28}$ H. Miyazaki, K. Tsukagoshi, A. Kanda, M. Otani, and S. Okada, Nano Lett. 10, 3888 (2010).

${ }^{29}$ M. Otani and S. Okada, J. Phys. Soc. Jpn. 79, 073701 (2010).

${ }^{30}$ Y. Sakai, T. Koretsune, and S. Saito, Phys. Rev. B 83, 205434 (2011).

${ }^{31}$ M. I. Katsnelson and A. K. Geim, Nat. Phys. 6, 30 (2009).

${ }^{32}$ V. M. Pereira and A. H. C. Neto, Phys. Rev. Lett. 103, 046801 (2009).

${ }^{33}$ D. A. Bahamon and V.M. Pereira, Phys. Rev. B 88, 195416 (2013).

${ }^{34}$ S. Souma, M. Ueyama, and M. Ogawa, Appl. Phys. Lett. 104, 213505 (2014). 
${ }^{35}$ J.-K. Lee, S. Yamazaki, H. Yun, J. Park, G. P. Kennedy, G.-T. Kim, O. Pietzsch, R. Wiesendanger, S. Lee, S. Hong, U. Dettlaff-Weglikowska,and S. Roth, Nano Lett. 13, 3494 (2013).

${ }^{36}$ H. Tomori, A. Kanda, H. Goto, Y. Ootuka, K. Tsukagoshi, S. Moriyama, E. Watanabe, and D. Tsuya, Appl. Phys. Express 4, 075102 (2011).

${ }^{37}$ P. Hohenberg and W. Kohn, Phys. Rev. 136, B864 (1964).

${ }^{38}$ W. Kohn and L. J. Sham, Phys. Rev. 140, A1144 (1965).

${ }^{39}$ Y. Morikawa, K. Iwata, and K. Terakura, Appl. Surf. Sci. 169-170, 11 (2001).

${ }^{40}$ J. P. Perdew, K. Burke, and M. Ernzerhof, Phys. Rev. Lett. 77, 3865 (1997).

${ }^{41}$ J. P. Perdew, K. Burke, and M. Ernzerhof, Phys. Rev. Lett. 78, 1396 (1997).

${ }^{42}$ D. Vanderbilt, Phys. Rev. B 41, 7892 (1990).

${ }^{43}$ G. Gui, J. Li, and J. Zhong, Phys. Rev. B 78, 075435 (2008).

${ }^{44}$ M. Farjam and H. Rafii-Tabar, Phys. Rev. B 80, 167401 (2009).

${ }^{45}$ G. Gui, J. Li, and J. Zhong, Phys. Rev. B 80, 167402 (2009).

${ }^{46}$ T. Miyake and S. Saito, Phys. Rev. B 68, 155424 (2003).

${ }^{47}$ T. Miyake and S. Saito, Phys. Rev. B 72, 073404 (2005). 